\title{
Santé et maladie des populations vulnérables
}

\section{Jean Martin}

Dr méd., membre de la rédaction

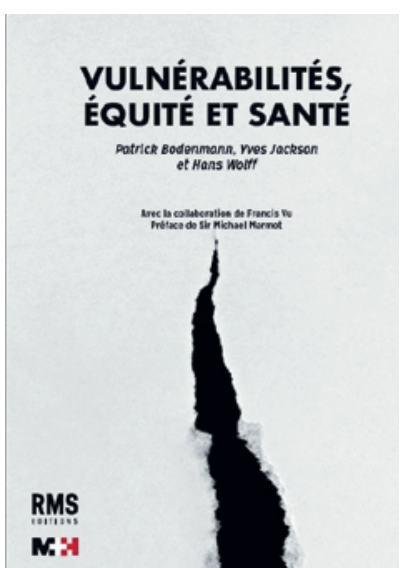

Patrick Bodenmann, Yves Jackson et Hans Wolff Préface de Sir Michael Marmot Vulnérabilités, équité et santé

Chêne-Bourg: Médecine et Hygiène; 2018. 430 pages. $45 \mathrm{CHF}$.

ISBN 978-2-88049-434-6

Pour planter le décor. Les soins à ceux qui, pour des raisons multiples, sont systématiquement (le mot n'est pas trop fort) précarisés du point de vue de leur santé, qu'on rassemble aujourd'hui sous le terme de populations vulnérables, cela n'est pas nouveau. Partie du mandat classique du médecin, qui traite chacun sans distinction de classe sociale ou de revenu - et fixe ses honoraires en fonction des conditions matérielles du patient (avant que les assurances universelles rendent - en principe - chacun solvable). Mais la réalité n'était pas toujours à l'image de la théorie... Les dernières décennies ont vu une "cristallisation" de ces préoccupations et l'émergence d'une clinique qui s'enracine sur les caractéristiques et besoins particuliers de ces groupes. Ceci en lien direct avec les acquis scientifiques de la santé publique quant aux inégalités/inéquités/écarts majeurs de santé au sein d'une même collectivité, quant aux déterminants sociaux en cause et au rôle de l'environnement de vie - magistralement étudiés au siècle dernier par le Britannique Th. McKeown (voir son An Introduction to Social Medicine de 1966), parmi d'autres. Ces réalités sont un fondement de l'action de l'OMS et doivent aujourd'hui être au premier plan des politiques de santé, partout (à l'évidence pas seulement dans les pays pauvres).*

Il importe de souligner que ces situations qu'il convient d'étudier et auxquelles il faut s'attaquer ressortissent à deux grandes problématiques: les inégalités constatées en tant que telles et les inégalités (systématiques elles aussi) dans l'accès aux soins.

Le livre. Vulnérabilités, équité et santé est publié sous la direction de P. Bodenmann, titulaire depuis 2016 de la Chaire de médecine des populations vulnérables (première du genre en Suisse) à la Policlinique médicale universitaire de Lausanne, $\mathrm{H}$. Wolff, chef du Service de médecine pénitentiaire de Genève, et Y. Jackson, de l'Institut de santé globale, Genève. Quatre parties comptant chacune une dizaine de chapitres. Chacun de ces derniers, avec des vignettes cliniques et de nombreuses références, bénéficie généralement d'une demi-douzaine de co-auteurs, pour un total de 120 auteurs - de Suisse en grande majorité. Dans l'introduction: "Ce livre a pour objectif de présenter les différents concepts de vulnérabilité en lien avec les inégalités de santé, dans un cadre ancré dans la pratique médicale. Il propose une revue des compétences requises et offre des conseils pratiques. Nous visons à sensibiliser les professionnels de la santé et du social à ces besoins spécifiques, y compris au plan éthique.» $\mathrm{A}$ noter la définition donnée de la précarité: «Un état d'instabilité sociale caractérisé par l'absence d'une ou de plusieurs sécurités, notamment celle de l'emploi, permettant aux personnes et familles d'assumer leurs obligations et de jouir de leurs droits fondamentaux.» Michael Marmot, une des figures actuelles de l'épidémiologie et de la santé publique (University College, Londres), a rédigé la préface. Extrait: «En cherchant à influencer les politiques, nous avons rencontré des problèmes. Premièrement, les médecins s'intéressent à la santé mais, dans l'ensemble, pas aux conditions qui mènent à la maladie. Le secteur de la santé, y compris les ministères, est centré sur les soins [...] Dans mon livre The Health Gap, je commence avec la question 'Pourquoi traiter les gens et les renvoyer dans les conditions qui les ont rendus malades?'» 
Un éventail de thèmes large et diversifié. Sans tenter de résumer une telle somme (au reste, les auteurs n'ont pas de prétention à l'exhaustivité), je cite des titres de chapitre: Médecine sociale et pratique clinique, quand la précarité précède la pauvreté - Littératie en santé Compétences cliniques transculturelles en pratique Ce que l'autre peut susciter chez vous (préjugés, stéréotypes) - Approches dans les soins de premier recours: pragmatisme lors de rencontres complexes - Migration sans frontières - Vieillir et devenir vulnérable Milieu pénitentiaire: vulnérabilité partagée entre détenus et professionnels de santé-Soins de qualité pour les personnes LGBT - Les grands consommateurs des services d'urgence - Besoins en santé des migrants, y compris enfants - Carie du biberon: un marqueur d'inégalités - Grève de la faim et détention - Maladies infectieuses chez les requérants d'asile et vaccinations - Renoncement aux soins: dépistage et prise en charge - Importance de la prise en compte de la spiritualité. Ci-dessous quelques éléments qui ont retenu mon attention.

Une approche pragmatique des situations interculturelles, proposée par J. Sanchis Zozaya et coll. (notamment en cas de troubles psychiques). Elle comporte dix questions, dont: 1) Quelles sont mes zones d'inconfort devant ce patient? - 2) Quelles représentations et attentes ont le patient et son entourage de la relation avec le professionnel de santé? - 3) Qu'est ce que le patient et son entourage pensent de la psychiatrie (respectivement, de la médecine)? - 5) Comment la souffrance se manifeste-t-elle selon sa culture? - 6) Quels sont mes doutes quant au diagnostic? - 8) Quels sont les enjeux autour de la langue maternelle et de la langue acquise (y compris interprétariat)?

Approche "syndémique». Je partage ma découverte de ce terme qu'on doit à l'anthropologue médical américain Merrill Singer, qui introduit les contextes social, politique et économique comme facteurs de maladies. Dans le chapitre sur la pandémie du syndrome métabolique, J. Ruiz et coll. écrivent: «L’approche syndémique met au même niveau l'environnement socioculturel, les comportements et la biomédecine et suggère une approche holistique. Elle propose aussi d'introduire d'autres partenaires de soins, tels les médiateurs culturels et travailleurs sociaux, pour avoir une approche centrée sur les racines du mal.» Noter que, en 2017, Lancet a publié une série sur la théorie syndémique. Là encore, on est au cœur de ce que souhaite/demande la santé publique.

Certificats médicaux. On sait les questions éthiques et pratiques autour de ces documents (où les valeurs du médecin peuvent entrer en contradiction avec les de- mandes de tierces personnes ou autorités). Pourtant, sous le titre "Pour une clinique du certificat» dans un chapitre sur les migrants, $M$. Saraga et coll. relèvent: «Le cas de l'aide d'urgence invite à reconsidérer la position largement répandue [que ce travail administratif détourne les médecins de leur activité propre]. La rédaction d'un rapport permettant d'améliorer les conditions de vie concrètes d'un patient peut aussi être envisagée comme un acte clinique. Que puis-je faire pour cette personne, quelle position puis-je prendre, qu'ai-je à apporter, comme médecin?»

Y aurait-il lieu de tolérer les inégalités de santé? Question interpelante posée par Samia Hurst. «Avec John Rawls notamment et au nom du principe de différence, il est devenu classique d'admettre qu'un degré d'inégalité est une bonne chose. En est-il ainsi des inégalités de santé? Clairement non. Espérer que des inégalités stimulent les efforts individuels pour la santé présuppose la liberté et les moyens de faire ces efforts. Or c'est précisément cela dont le manque soutient les inégalités que l'on constate.» A noter qu'elle propose de définir la vulnérabilité comme «une probabilité accrue de subir un tort: n'importe quel tort, pour n'importe quelle raison".

En guise de conclusion. Dans la post-face du Dr S. Spycher, Vice-directeur de l'OFSP: «Le thème de l'équité des soins en Suisse est inconfortable pour beaucoup; il est même inimaginable en raison de notre richesse [...] L'OFSP prend cette question très au sérieux. Notre compréhension du besoin d'agir est que nous ne pouvons pas améliorer la qualité de soins sans en améliorer l'équité. [Ceci] relève d'une responsabilité partagée des différents acteurs.»

Vulnérabilités, équité et santé comble à l'évidence une lacune. Il existe des ouvrages de santé publique et communautaire mais pas jusqu'ici, chez nous, de traité qui établisse de manière substantielle et diversifiée le lien avec la clinique quotidienne, toujours complexe, auprès des populations vulnérables. Son poids (un kilo!) ne permet guère de l'avoir dans la poche, mais ne l'empêche pas d'être un instrument de référence qu'on aura près de soi. Pour le consulter sur les questions et défis de la relation avec et la prise en charge de personnes et groupes qui sont à l'écart: à cause de leur manque d'insertion dans le «mainstream», à cause (cf. supra) de «l'absence d'une ou plusieurs sécurités», avec une "probabilité accrue de subir un tort». Les professionnels francophones sont ainsi chanceux de disposer de cette somme - dont on souhaite, pour ce qui concerne notre pays, qu'elle puisse bénéficier d'une traduction allemande. 